\title{
Coding Assisted Adaptive Noise Cancellation in Stochastic MIMO Wireless Channels
}

\author{
Subhadra Das \\ Department of Electronics and Communication \\ Engineering \\ GIMT, Azara , Hathkhowapara, Guwahati-781017
}

\author{
Kandarpa Kumar Sarma \\ Department of Electronics and Communication \\ Engineering, \\ Gauhati University, Guwahati-781014, Assam, \\ India.
}

\begin{abstract}
Fading is a random behavior and generally modeled with statistical distributions. Fading caused by multipath propagation can degrade the bit error rate (BER) performance of a digital communication system. To mitigate fading and to have reliable communication in wireless channel error control coding along with adaptive filtering techniques are employed. In this paper, certain methods of noise cancellation in stochastic multiple-input-multiple output wireless channel using error correction coding as well as adaptive filter trained with Least Mean Square (LMS), Normalised Least Mean Square (NLMS) and Recursive Least Square (RLS) algorithm are explored. The experiments performed show satisfactory results in severely faded Nakagami-m channels for SIMO, MISO and MIMO set ups. The work intends to formulate a framework for developing certain insight into the use of error control coding and adaptive filtering to fight fading in stochastic wireless channel.
\end{abstract}

\section{General Terms \\ Algorithms}

\section{Keywords}

Adaptive filters, Nakagami-m fading, convolutional code, SIMO, MISO, MIMO System.

\section{INTRODUCTION}

Transmission of information in real world communication system is effected by noise and is thus susceptible to errors. A practical option for changing the data quality is error control coding. Error control coding is used with data transmission over noisy communication channel. Again, adaptive filters have the ability to adjust their own parameters automatically and in their designing no prior knowledge of signal or noise characteristics [1] [2]. Real-time adaptive filtering algorithms and error correction coding techniques are essential components of most present-day communications in both wired and wireless forms.

In this paper, certain methods of noise cancellation in stochastic multiple-input-multiple output wireless channel using error correction coding as well as adaptive filter trained with Least Mean Square (LMS), Normalised Least Mean Square (NLMS) and Recursive Least Square (RLS) algorithm have been explored. The experiments performed show satisfactory results in severely faded Nakagami-m channels for SIMO, MISO and MIMO set ups. The work intends to formulate a framework for developing certain insight into the

use of error control coding and adaptive filtering to fight fading in wireless channel.

\section{THEORETICAL BACKGROUND}

In this section, the basic theoretical notions essential for the work have been briefly described

\subsection{Error Control Coding}

The technique used for controlling errors in data transmission over noisy communication channel is error control coding. Forward error correction is accomplished by adding redundancy to the transmitted information using a predetermined algorithm [3]. Figure 1 below depicts a trellis for a convolutional encoder

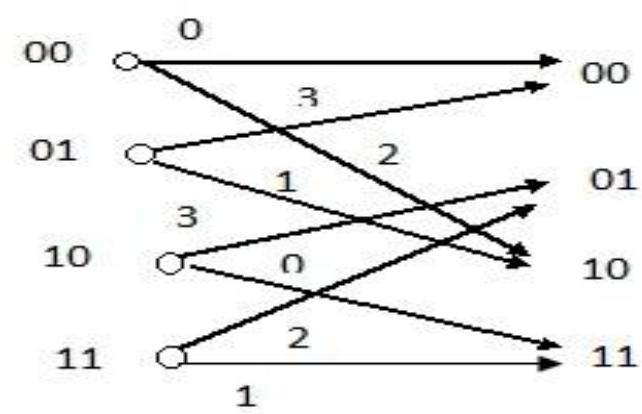

Figure 1. Trellis for a convolutional encoder

\subsection{Adaptive Filter}

An adaptive filter consists of two basic elements [2]:-

- Digital filter, which produces an output in response to an input signal.

- An adaptive algorithm, which adjusts the coefficients of the digital filter.

Adaptive filter block diagram is shown in Figure 2. The signal $\mathrm{d}(\mathrm{n})$ is called the desired signal. The input and the output of the filter are denoted by $\mathrm{x}(\mathrm{n})$ and $\mathrm{y}(\mathrm{n})$ respectively. The signal $\mathrm{e}(\mathrm{n})$ is called the estimation error and is defined by $\mathrm{e}(\mathrm{n})=\mathrm{d}(\mathrm{n})$ $y(n)$. The adaptive algorithm is so designed that it minimizes some objective function contributed from this error signal.

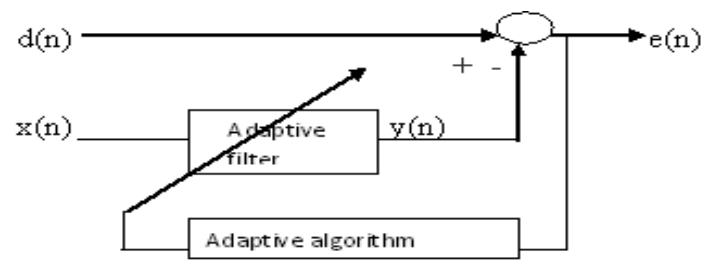

Figure 2. Adaptive Filter Block Diagram 


\subsection{Adaptive Noise Cancellation}

Basically an adaptive noise canceller is operated on the outputs of two sensors:

- A primary sensor that supplies a desired signal of interest effected by noise

- A reference sensor that supplies noise alone.

The signal and noise components at the output of the primary sensor are uncorrelated and the noise at the output of the reference sensor is correlated with the noise component of the primary-sensor output. The adaptive noise canceller consists of an adaptive filter that operates on the reference sensor output to produce an estimate of the noise, which is then subtracted from the primary sensor output. The overall output of the canceller is used to control the adjustments applied to the tap weights in the adaptive filter. The adaptive canceller minimizes the mean-square error (MSE) value of the overall output, thereby causing the output to be the best estimate of the desired signal in the minimum-mean-square error sense [1]. The block diagram of dual input adaptive noise canceller is shown Figure 3.

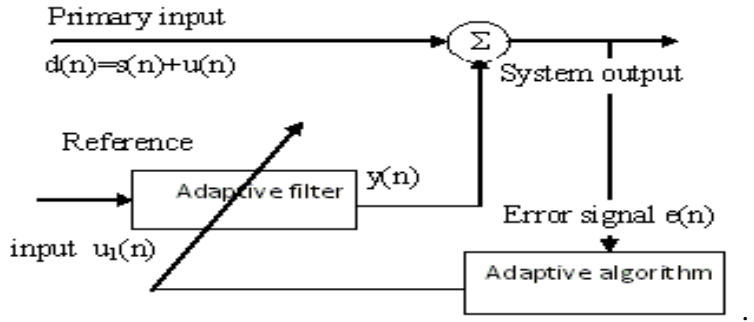

Figure 3. Dual Input Adaptive Noise Canceller

\subsection{Stochastic Wireless Channels}

Transmitted signals in wireless communications are associated with a physical phenomenon called fading. With the hypothesis of many mathematical models used to model fading, Nakagami fading channel model has been widely adapted. Nakagami fading provides a generalized system for analysis and design and simplifies conversion between different stochastic behavior. It is reliable when compared to other fading models like Rayleigh and Log-normal [5]. Also, Nakagami $\mathrm{m}$ distribution gives the best fit to some urban multipath data.

The Nakagami m distribution is given by

$$
f(x)=\frac{2}{\Gamma(m)}\left(\frac{m}{P}\right)^{m} \cdot x^{2 m-1} \cdot \exp \left(-\frac{m}{P} x^{2}\right)
$$

where $ґ()=$. gamma function .

$$
\begin{aligned}
& \mathrm{P}=\text { average signal power. } \\
& \mathrm{m}=\text { fading parameter. }
\end{aligned}
$$

For $m=1$, the Nakagami-m distribution reduces to a Rayleigh distribution and for $1<\mathrm{m}<2$, the Nakagami- $m$ distribution tends to a Rician distribution. The probability density function of Nakagami-m fading channel is given in figure 4 [6]. As a result arrangements like multiple-input-single output system and multiple-input-multiple-output systems are obtained from the basic single-input-single-output set up. Alamouti space time block code (STBC) is an attractive technique to achieve diversity performance in MIMO system. It is assumed that the system contains two transmit antennas and one receive antenna for MISO system and two transmit and two receive antennas for MIMO system. At the transmitter the data of two consecutive slot will be considered. At the odd time slot, the first antenna transmits symbol $1\left(\mathrm{~s}_{1}\right)$ and the second ones will transmit symbol $2\left(\mathrm{~s}_{2}\right)$ simultaneously. At the even time slots, $-\mathrm{s}_{1}{ }^{*}$ and $\mathrm{s}_{1}{ }^{*}$ will be transmitted [8]. A narrowband flat fading MIMO system is modeled as

$$
y=H x+n
$$

where $\mathrm{y}$ and $\mathrm{x}$ are receive and transmit vector, $\mathrm{H}$ and $\mathrm{n}$ are channel matrix and noise vector. A MIMO system model is shown in figure 5 .

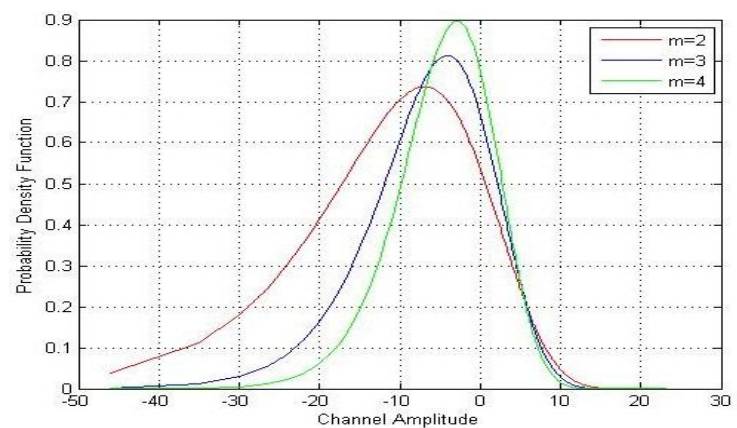

Figure 4. Probability Density Function for Nakagami- m distribution for different $m$ values.

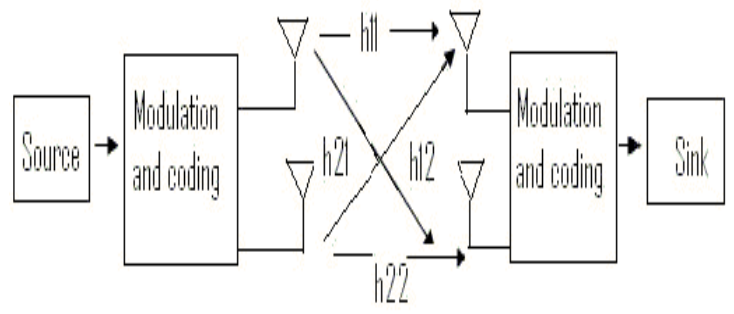

Figure 5. MIMO System model.

\subsection{Diversity}

An effective way of achieving transmit diversity is by the method of space time block coding. The Alamouti scheme is a method of obtaining transmit diversity for the case of two transmit antennas. Two symbols are considered at a time say $x_{1}$ and $x_{2}$ and they are transmitted in two consecutive time slots. In the 1st time slot, $x_{1}$ is transmitted and the 2 nd time slot $x_{2}$ is transmitted from the 2 nd one. In the 2 nd time slot,$\mathrm{x}_{1}{ }^{*}$ is transmitted from 1 st antenna, while $-\mathrm{x}_{2}{ }^{*}$ transmitted from 2 nd antenna. The signals $-\mathrm{x}_{1}{ }^{*}$ and $x 2$ are picked from an arbitrary M-ary constellation. Since, symbols are transmitted in two time slots, overall transmission rate is 1 symbol per channel [9].

The Alamouti scheme is shown in figure 6 .

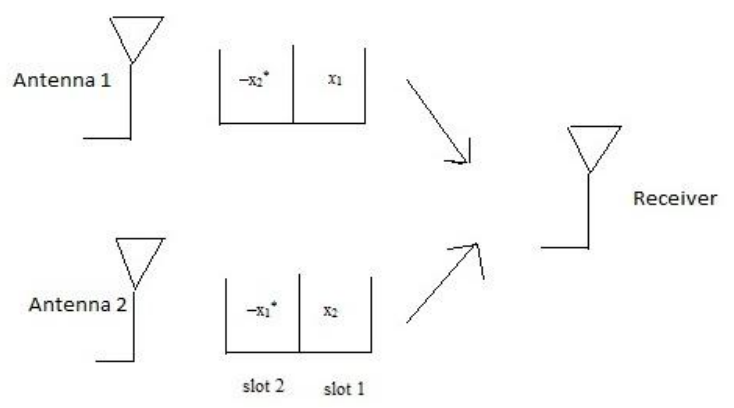

Figure 6. Alamouti Scheme 


\section{NOISE CANCELLATION IN STOCHASTIC MIMO WIRELESS CHANNELS USING CODING AND ADAPTIVE FILTERING : EXPERIMENTAL \\ CONSIDERATIONS}

Here, some noise cancellation experiments are described which have been performed using error correcting convolutional codes and adaptive filter trained with LMS, NLMS and RLS algorithms in single-input-single-output (SIMO), multiple-input-single-output (MISO) and multipleinput-mulitple-output (MIMO) set ups. Nakagami-m distribution is used to generate the fading channel characteristics. The corresponding BER curves are plotted for all the cases

\subsection{Noise Cancellation using error control coding}

Here, convolutional code has been used where the encoding operation can be viewed as the discrete time convolution of the input sequence with the impulse response of the encoder. A convolutional coder generates redundant bits by using modulo-2 convolution. A binary convolution code is generated by passing the information sequence to be transmitted through a linear finite state shift register. For a $(\mathrm{n}, 1, \mathrm{~K})$ convolutional code, the shift register consists of $\mathrm{K}$ stages and $n$ linear modulo- 2 function generators [4]. The input data is shifted into and along the shift register a single bit at a time producing a n-tuples output for each shift. The code rate of a $(n, 1, K)$ convolutional encoder is $1 / n$. The Viterbi algorithm is used as the decoding method for convolutional code [4]. It is a maximum likelihood decoding algorithm which upon receiving the channel output, searches through the trellis diagram to find the path that is most likely to have generated the received sequence. Here, the performance of a binary communication system that uses convolutional code with binary phase shift keying modulation is shown. It shows the error correcting capability of the code. The Viterbi Algorithm is used as the decoding method for convolutional codes.

\subsection{Adaptive Noise Cancellation in Nakagami-m Fading channel with 1x2 SIMO, 2x1 MISO and 2x2 MIMO system}

Adaptive filters trained with LMS, NLMS and RLS algorithm initially track variations in Nakagami-m fading channel and perform noise cancellation. A complex, uncorrelated white Gaussian noise with zero mean and unit variance is generated and added to the channels. The adaptive filter operates on the noise corrupted signal and produces an estimate of the noise, which is subtracted from the desired signal [10]. The overall output is used to control the adjustments applied to the tap weights in the adaptive filter.

\section{SIMULATION RESULTS AND DISCUSSION}

The experimental process logic is depicted by a flowchart shown in Figure 7 . The results were obtained using binary phase shift keying (BPSK) modulation along with convolutional coding[11]. The Nakagami-m fading envelope has a background noise which is additive Gaussian. Adaptive filter trained with LMS, NLMS and RLS algorithms initially track variations in faded Nakagami-m channel and perform noise cancellation. Adaptive filtering with convolutional coding is applied in 1x2 SIMO, 2x1 MISO and 2x2 MIMO systems[12]. The SNR vs BER plot are shown using adaptive algorithms in Nakagami-m fading filled SIMO, MISO and MIMO system with and without coding in figures 8,11 and 12.

The estimated waveforms using LMS, NLMS and RLS algorithm with and without coding in SIMO, MISO and MIMO system shown in figure 9,10 and 13.The coding gain calculated for SIMO, MISO and MIMO system with and without coding and is shown in table 1.

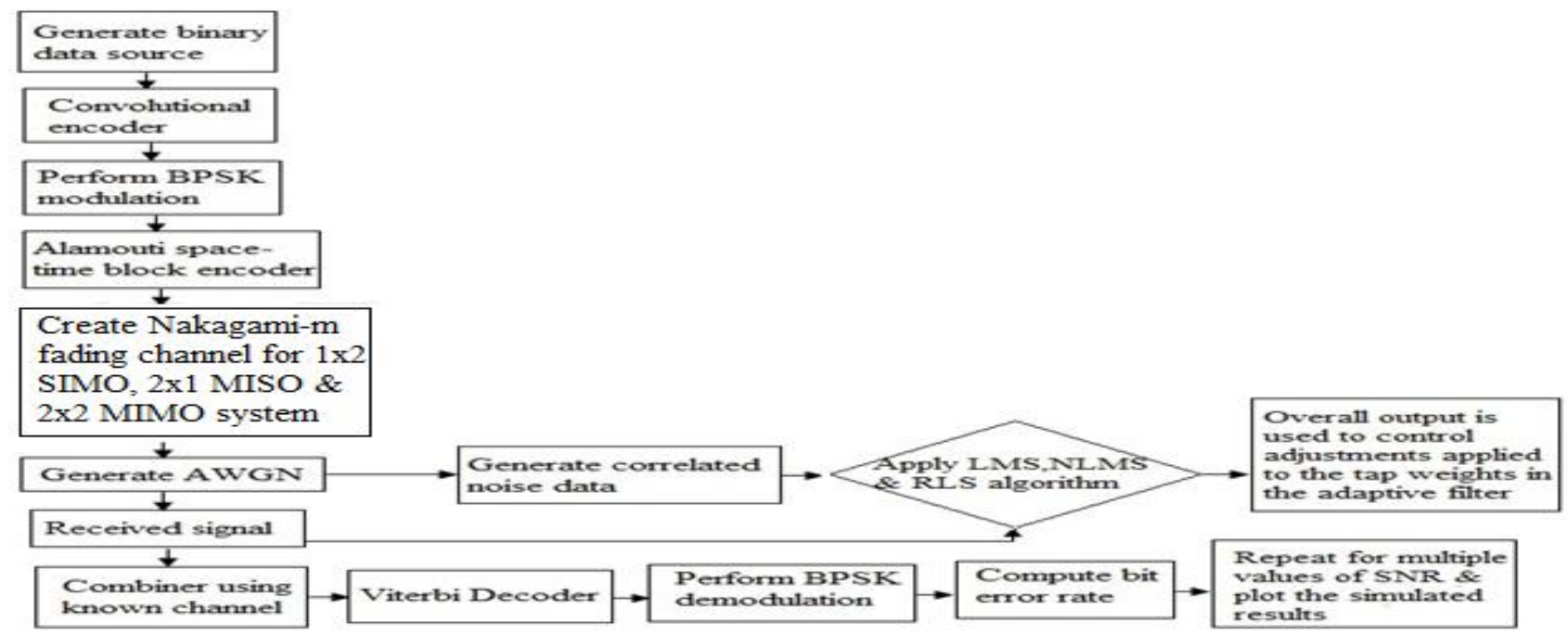

Figure 7: Flowchart depicting the process logic using adaptive filters and convolutional coding with multiple antenna systems. 


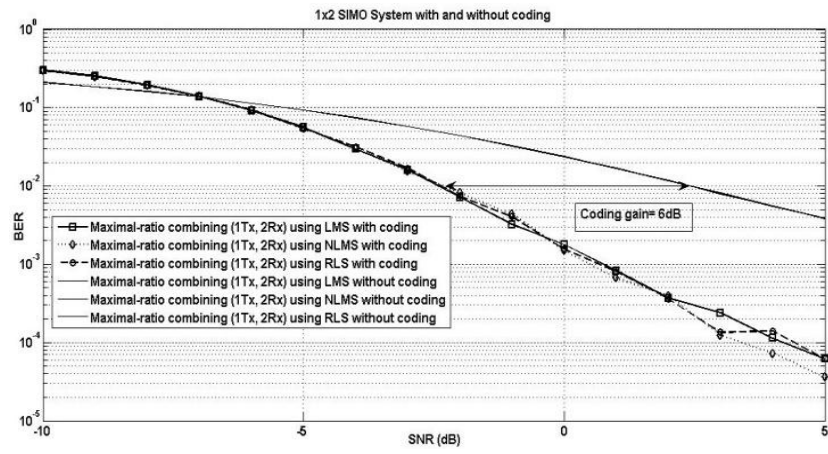

Figure 8. SNR vs BER plot for BPSK modulation with and without convolutional coding with $1 \times 2$ SIMO System

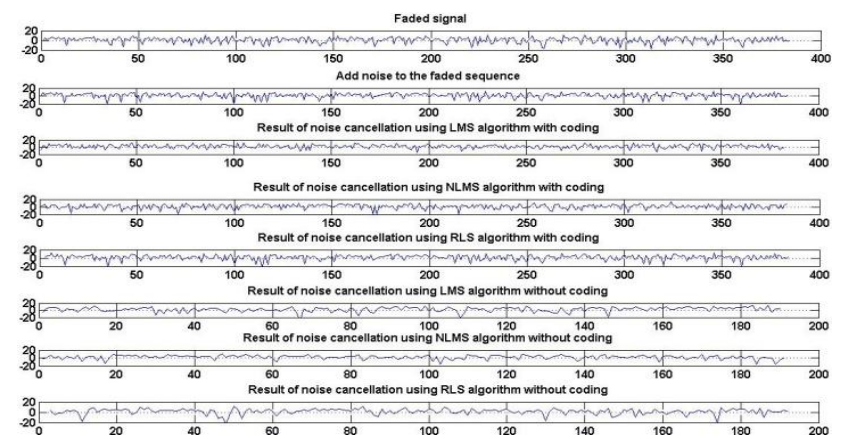

Figure 9. Adaptive Noise Cancellation for BPSK Modulation with and without coding in 1x2 SIMO system

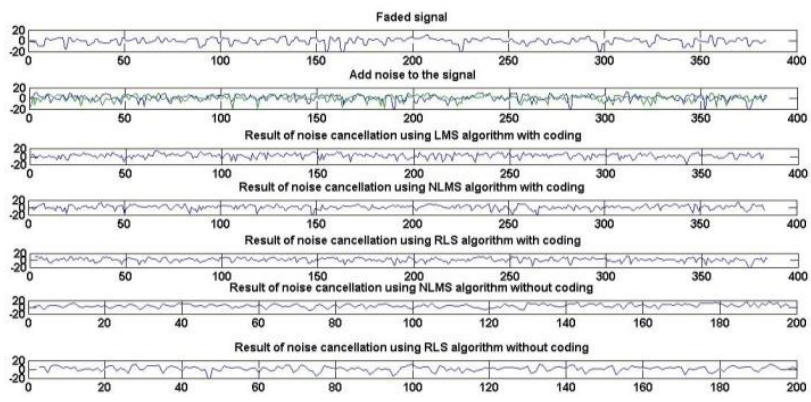

Figure 10. Adaptive Noise Cancellation for BPSK modulation with and without convolutional code with $2 \times 1$ MISO system

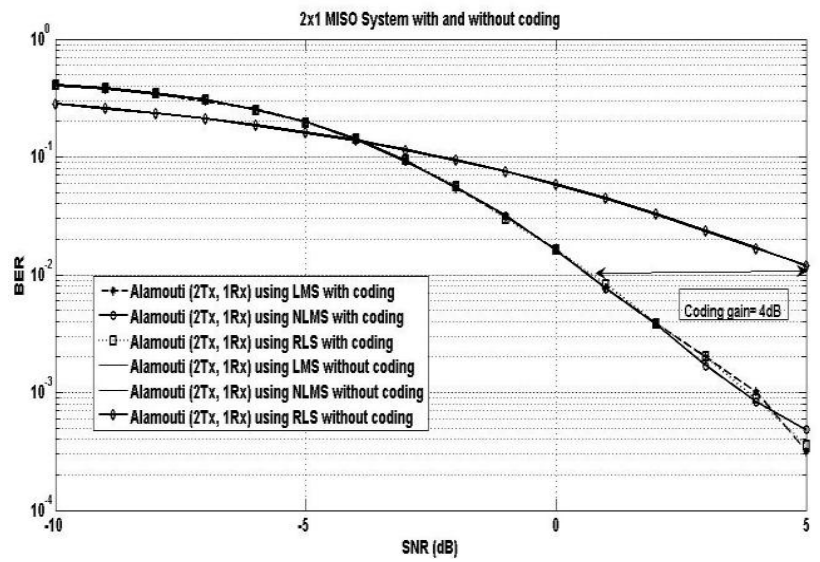

Figure 11. SNR vs BER plot for BPSK modulation with and without convolutional coding with $2 \times 1$ MISO system

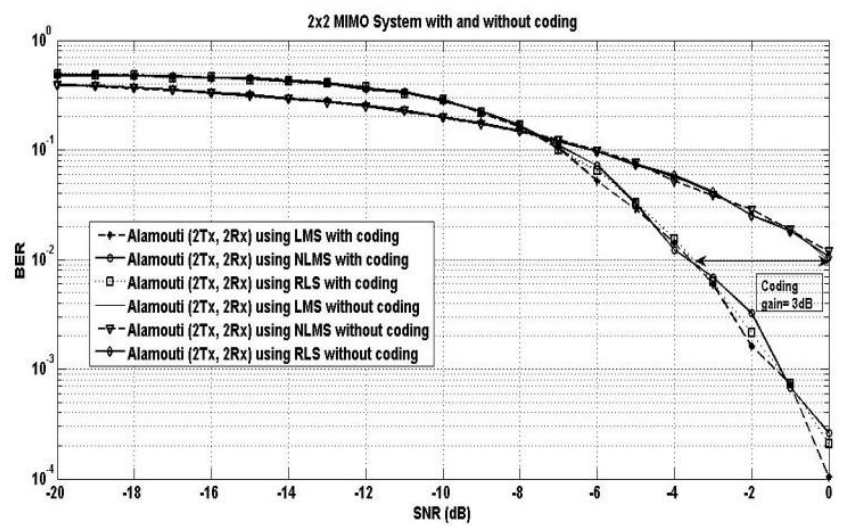

Figure 12. SNR vs BER plot for BPSK modulation with and without convolutional coding for $2 \times 2$ MIMO system

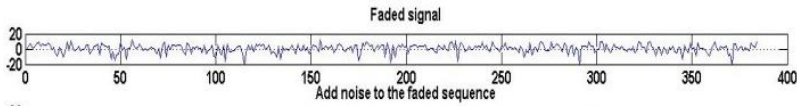

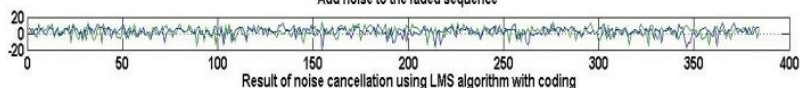

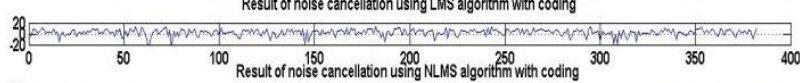

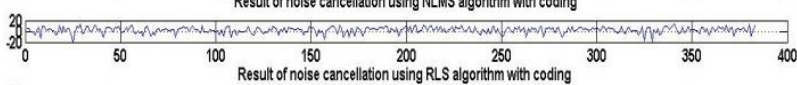

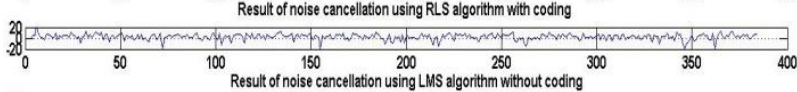
Result of noise cancellation using LMS algorithm without coding

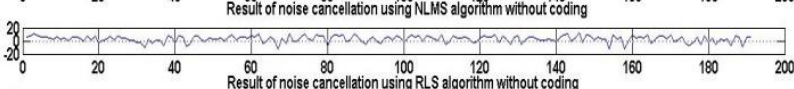
28 Result of noise cancellation using RLS algontinm without cooing

Figure 13. Adaptive Noise Cancellation for BPSK modulation with and without convolutional coding with 2x2 MIMO system

Table 1. Coding Gain for SIMO, MISO and MIMO System using BPSK modulation

\begin{tabular}{|l|l|l|l|l|}
\hline Scheme & $\begin{array}{l}\text { BER } \\
\text { values }\end{array}$ & $\begin{array}{l}\text { SNR } \\
\text { coded }\end{array}$ & $\begin{array}{l}\text { SNR } \\
\text { uncoded }\end{array}$ & $\begin{array}{l}\text { Coding } \\
\text { gain }(\mathbf{d B})\end{array}$ \\
\hline SIMO & $10^{-2}$ & -3 & 3 & 6 \\
\hline MISO & $10^{-2}$ & 1 & 5 & 4 \\
\hline MIMO & $10^{-2}$ & -3 & 0 & 3 \\
\hline
\end{tabular}

With LMS, NLMS and RLS algorithms, 2x2 MIMO system with coding provides better performance and on average, provides $3 \mathrm{~dB}$ coding gain in severe faded channels. The framework thus formulated provides satisfactory results while carrying out noise cancellation using adaptive algorithms and using convolutional coding in SIMO, MISO and MIMO systems. The use of adaptive filtering and error control coding depicts the approach to fight fading in wireless channel. The proposed model gives better results to combat fading compared to the work done in [12]

\section{CONCLUSION}

Here, a framework for noise cancellation using error control coding as well as adaptive filter trained with LMS, NLMS and RLS algorithm in severely faded Nakagami-m channel with 
SIMO, MISO and MIMO systems has been proposed. The experimental results show satisfactory performances and a simplistic arrangement to tackle noise related variations in the channel. With LMS, NLMS and RLS algorithms, 2x2 MIMO system with coding provides better performance and on average, provides $3 \mathrm{~dB}$ coding gain in severe faded channels.

The work can be extended to an adaptive mechanism using real time capabilities for which Kalman filters can be used. Also some modulation schemes can be used of higher order to achieve better performance. Further, the approach will be considered to propose a system which deals with a joint venture of source coding and channel coding to tackle fading in wireless channels along with diversity considerations.

\section{REFERENCES}

[1] B. Widrow, J. R.Glover, J. M.McCool, J. Kaunitz, C. S. Williams, R. H. Hearn, J. R. Zeidler,Eugene Dong, R. C. Goodlin: "Adaptive Noise Cancelling: Principles and Applications," Proceedings of the IEEE, vol. 63, no. 12, Dec. 1975.

[2] S. Haykin, “Adaptive Filter Theory”, $4^{\text {th }}$ ed., PrenticeHall, Inc., 1991.

[3] T. S. Rappapot, "Wireless Communication", $2^{\text {nd }}$ ed., Prentice-Hall, Inc.,2002.

[4] S. Haykin,"Communication Systems", 4th ed., WileyIndia Inc., 2001

[5] G.S.Prabhu, P.M. Shankar, "Simulation of Flat Fading Using MATLAB for Classroom Instruction”,Department of Electrical and Computer Engineering,Drexel University,3141 Chestnut Street,Philadelphia, PA 19104.

[6] N. Srivastava, A. Johri , M.Saxena, "Performance of Nakagami-m fading channel with equal gain combining and maximan ratio combining", International Journal of Electronics Communication and Computer Engineering, Vol. 3, Issue 1, 2012

[7] H. Kaur, "Applications and Simulation of Adaptive Filter in Noise Cancellation, June 2011.

[8] O. B. Sik, C. Cardinal, F. Gagnon, "On the Performance of Interference Cancellation in Wireless $\mathrm{Ad} \mathrm{Hoc}$ Networks," 2010 IEEE.

[9] S.Catreux,P.F.Driessen,L.J.Greenstein, "Simulation results for an interference-limited multiple input multiple output cellular system," Vol. 2 , 2000.

[10] M. H.Hayes, "Statistical Digital Signal Processing and Modelling”,Wiley-India.

[11] S.Das, K.K. Sarma, "Noise Cancellation in Stochastic Wireless Channels using Coding and Adaptive Filtering", Communicated to International Journal of Computer Applications (IJCA).

[12] S. Das, K. K. Sarma, "Noise Cancellation in Stochastic Wireless Channels using Adaptive Filtering", Proceedings of International Conference on Electronics and Communication Engineering (ICECE), pp. 23-28 Guwahati, India, Jan.14, 2012 